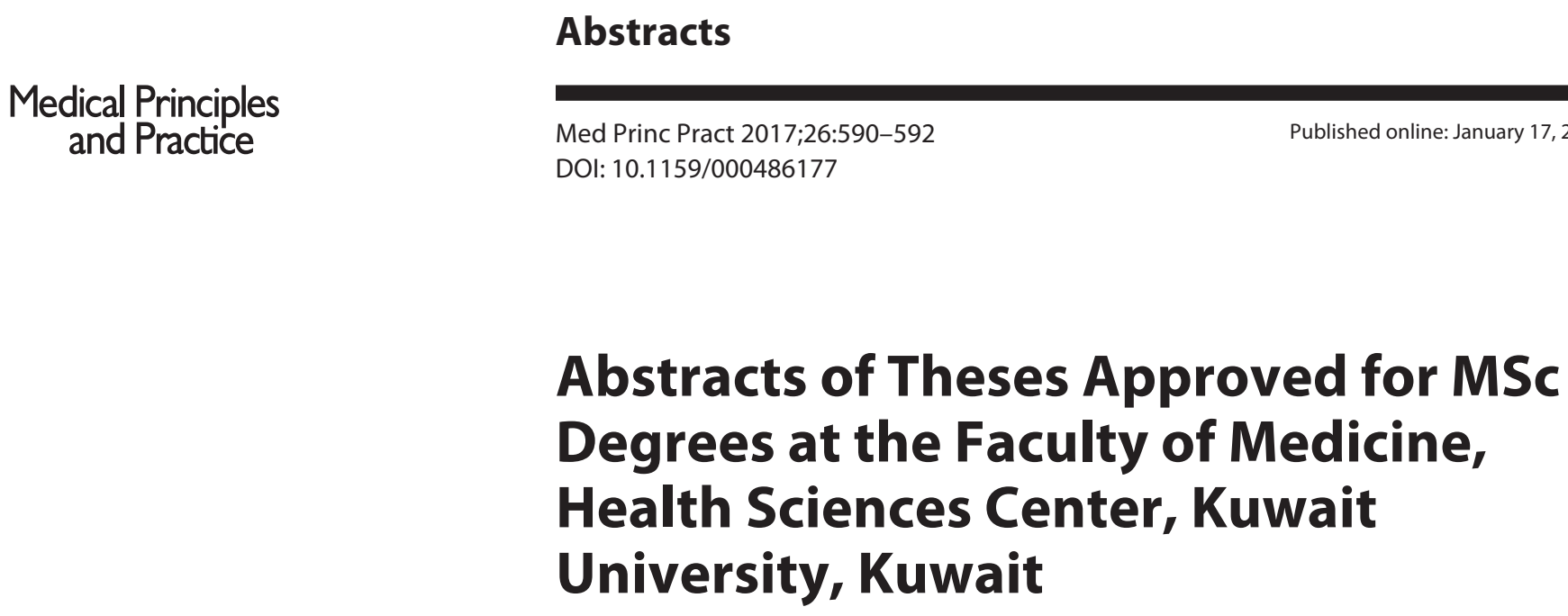

\section{MSc Degree}

\section{1}

\section{Cytotoxic Activities of Mn(III) N-Substituted} Alkylpyridylporphyrins

\section{Bader H.R. Bader}

Department of Biochemistry, Faculty of Medicine, Kuwait University, Kuwait

Manganese porphyrins ( $\mathrm{MnP})$ are superoxide dismutase synthetic mimetics that accumulate within cancer cells along with ascorbate and generate hydrogen peroxide when combined. MnP differ in their reduction potential, lipophilicity, and bulkiness, which are parameters that affect cellular uptake, subcellular distribution, and the rate of hydrogen peroxide production. The aim of this thesis was to use a panel of MnPs with different peripheral substituents to investigate how structural modifications that alter the reduction potential, lipophilicity, and size of the $\mathrm{MnP}$ affect the cytotoxic and antiproliferative effects in vitro of different MnPs, either alone or in combination with ascorbate, on 2 human breast cancer cell lines which differ in multidrug resistance (PII, MDAMB-231) and a nontumorigenic human breast cell line (HBL-100). PII and HBL-100 displayed a similar sensitivity to ascorbate-induced cytotoxicity while MDA-MB-231 was more resistant. MnP and ascorbate displayed limited cytotoxicity to all cell lines but major cytotoxicity was seen with combinations of 1-5 $\mu \mathrm{M}$ MnTE$2-\mathrm{PyP}$ or MnTE-3-PyP and $1 \mathrm{~mm}$ ascorbate. The removal of exogenous $\mathrm{MnP}$ or addition of catalase to the medium prevented cytotoxicity, suggesting that hydrogen peroxide in the extracellular medium is responsible for cytotoxicity. Studies of cellular prolif- eration $(72 \mathrm{~h})$ displayed cytotoxic and cytostatic effects, and findings indicated conditions for the potential selectivity for cancer cells ( $1 \mu \mathrm{M} \mathrm{MnP}$ and $1 \mathrm{mM}$ ascorbate). Plasma membrane integrity was lost and cells displayed apoptotic markers after treatment with $5 \mu \mathrm{M}$ MnTE-2-PyP and $1 \mathrm{mM}$ ascorbate (4 h). Immunoblot analysis (caspase $7 / 9$, cleaved PARP) suggested caspase-independent cell death. The reduction potential of $\mathrm{MnP}$ is the most important parameter for MnP-ascorbate-induced cytotoxicity.

Prof. Ludmil Benov (Supervisor)

Dr. James Craik (Co-Supervisor)

Studies of Aldosterone Synthase-Antibody-Reactive Proteins in the ACTH-Stimulated Adrenal Gland

Inchirah N. Iskandarani

Department of Biochemistry, Faculty of Medicine, Kuwait

University, Kuwait

Rat adrenocortical tissue is organized into 3 concentric zones: glomerulosa (ZG), fasciculata (ZF), and reticularis (ZR). ZG expresses aldosterone synthase (CYP11B2) to synthesize aldosterone, whereas $\mathrm{ZF}$ and $\mathrm{ZR}$ produce corticosterone. Our recent studies showed that the stimulation of rats with ACTH for 4 consecutive days resulted in elevated corticosterone production, but also with sustained aldosterone synthesis. The hormone action did not affect the CYP11B2 protein level but induced a $68-\mathrm{kDa}$ protein (referred to as AAA-protein), detected by Western blot using the same antiCYP11B2 antibody. The present research was designed to carry out: (i) further characterizations of CYP11B2 and the AAA-protein, and (ii) exploration of whether ACTH also upregulates the adrenal mineralocorticoid receptor (MCR) protein level. The outcomes are

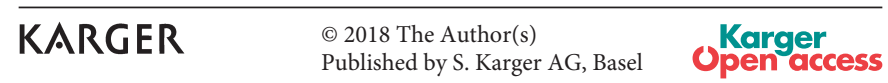

This is an Open Access article licensed under the Creative Commons Attribution-NonCommercial-4.0 International License (CC BY-NC) (http://www.karger.com/Services/OpenAccessLicense), applicable to the online version of the article only. Usage and distribution for commercial purposes requires written permission. 
noteworthy. Firstly, the cells immunostained for CYP11B2 primarily reside in ZG. Strikingly, ACTH stimulation causes an intensive appearance of the cells throughout ZF and ZR, but less so in ZG. Since ACTH action reportedly suppresses CYP11B2 gene expression, the altered distribution may be a consequence of the accelerated migration of the cells into the inner cortex. Secondly, ACTH stimulation upregulates the adrenal MCR protein level by $68 \%$. The treatment of rats with dexamethasone downregulates the protein level. The cells immunostained for MCR predominantly reside in ZG, but the cells become scattered throughout the inner cortex upon hormone stimulation. Thirdly, subcellular fractionation studies reveal that MCR and its guardian $11 \beta$-hydroxysteroid dehydrogenase-II are both localized in the microsomal fraction. ACTH-stimulation does not result in the translocation of MCR into the nuclear fraction, suggesting that the MCR-mediated aldosterone effect might be nongenomic. Unexpectedly, a subfraction of CYP11B2 (but not CYP11B1 and cytochrome oxidase) is observed in the cytosol, although they are all mitochondrial enzymes. Finally, study of the AAA-protein by 2 different anti-CYP11B2 antibodies does not entirely validate the previous observation. Thus, this protein was not considered further. The above data support the hypothesis that aldosterone may act as an autocrine/paracrine to regulate glucocorticoidogenesis through a synchronized mechanism by which CYP11B2-expressing cells and an increased MCR protein level are both made available in the inner adrenal cortex to cope with repetitive ACTH stimulation.

Dr. Behling Cheng (Supervisor)

Dr. Narayana Kilarkaje (Co-Supervisor)

\section{3 \\ Hormonal Influences on Cytokine Production Patterns in Unexplained Recurrent Spontaneous Miscarriage}

Ghadeer Jasiem Abdul Hussain

Department of Microbiology, Faculty of Medicine, Kuwait University, Kuwait

Maternal-fetal immunological interactions in general, and the cytokine milieu in particular, have been deemed critical to ensure feto-maternal tolerance for a successful pregnancy. Several lines of evidence support the notion that maternal anti-inflammatory Th2 cytokines promote successful pregnancy while pro-inflammatory Th1 cytokines are associated with pregnancy loss. With newly identified cytokines, the initial Th1/Th2 hypothesis has been expanded to Th1/Th2/Th17. Studies have reported higher levels of inflammatory cytokines in women who experience unexplained recurrent spontaneous miscarriage (uRSM) than in women who have a healthy pregnancy. The possible nexus between uRSM and maternal inflammatory bias has opened up the possibility of using hormones such as progesterone and dydrogesterone to alter the maternal cytokine bias in a manner that is conducive to successful pregnancy. The objective of this thesis was to investigate the ability of progesterone, dydrogesterone, and estrogen to modulate cytokine production by peripheral blood lymphocytes from women undergoing uRSM. Our study provides new findings that, in addition to Th1 cytokines, mitogen-activated maternal PBMCs of women with uRSM produce elevated levels of Th17 cytokines, in- dicating that elevated Th17 immunity is also involved in the pathogenesis of uRSM. Additionally, the data presented in this thesis indicate that progesterone, dydrogesterone, and estrogen are important regulators of Th1/Th2/Th17 immunity capable of modulating pro- and anti-inflammatory cytokine production in uRSM, creating a milieu that is conducive to the success of pregnancy. The results shed light on the roles of cytokine networks in the fetalmaternal relationship and on immunomodulation using pregnancy-related hormones that may provide viable treatment options for patients with unexplained recurrent miscarriage.

Prof. Raj Raghupathy (Supervisor)

\section{4}

Rat Astrocytes and Brain Pericytes: Secretome Profiles of Cytokines and Chemokines during Anoxia

Zeinab Adel Mahmoud Samy Sayed

Department of Physiology, Faculty of Medicine, Kuwait

University, Kuwait

Cerebral ischemia occurs when cerebral blood flow is reduced to a point at which it is not sufficient to sustain local metabolism, which causes functional impairment and could lead to brain tissue damage. Protective or detrimental effects of several cytokines and chemokines that are released from the cells of the neurovascular unit during cerebral ischemia are well documented. However, no study so far has explored the secretome profile of a large panel of these signaling molecules. The aim of this in vitro study was to use the advantages of a recently developed multiarray assay in order to explore the time patterns of secretion of 30 cytokines and chemokines from rat brain astrocytes and pericytes in primary cultures during anoxia and to compare these to the corresponding pattern of secretion in the presence of oxygen. Primary cultures were produced and exposed either to normoxia or anoxia for 2-48 h. After exposure, the conditioned medium was harvested and processed for profiling of cytokines and chemokines using a rat cytokine array panel. In addition, the time pattern of angiopoietin- 1 secretion was determined by ELISA. Changes in the secretion of cytokines and chemokines from astrocytes during anoxia were related to a particular molecular network by importing the data into the core analysis tool of Ingenuity Pathways Analysis (IPA) software. The main findings were that astrocytes secreted all tested cytokines at various time points even in the presence of oxygen. During anoxia, secretion of these cytokines is largely increased, although different time patterns were revealed for different cytokines. In contrast to astrocytes, pericytes released 20-26 cytokines in the presence of oxygen, while in anoxia this secretion was severely reduced. A striking observation was that concentrations of most cytokines were higher in the medium harvested from pericytes (regardless of the conditions) than in the medium harvested from astrocytes. IPA analysis revealed that the main pathway that was triggered in astrocytes by anoxia was the HIF-1 $\alpha$-mediated pathway. However, 3 other pathways that are related to antigen presentation and inflammation were also triggered in astrocytes. Thus, it appears that astrocytes play an important role in triggering and regulating inflammation in cerebral hypoxia/ischemia.

Prof. Zoran Redzic (Supervisor)

Dr. Marian Turcani (Co-Supervisor) 


\section{5}

\section{Contractility and Motility of Rat Brain Pericytes under Normal Conditions and during Anoxia in vitro}

Lulwa Abdulateef Abdullah Dawood Al-Abdullah

Department of Physiology, Faculty of Medicine, Kuwait

University, Kuwait

In vivo evidence indicated that stroke triggers a rapid death of brain pericytes and a prolonged contraction of their processes, which impairs blood flow following reperfusion of the occluded artery. Other in vivo studies have challenged this hypothesis. In this study, we cultured brain pericytes from Sprague-Dawley rats, verified the purity of the cultures by immunostaining, and assessed their viability and contractility before and after exposure to oxygen glucose deprivation (OGD), which is an in vitro condition that partially mimics conditions that occur during stroke. The rationale of the method used was that a contraction of pericytes would inevitably reduce their movements in culture and would shorten the length of the cellular processes. The parameters were assessed by cell culture imaging in the cell observer for $15 \mathrm{~h}$, first before and then after OGD protocols and the subsequent analysis of images by SynoQuant software. Single cell membrane mobility (SCMM), the area/perimeter $(\mathrm{A} / \mathrm{P})$ ratio, and cell complexity, the latter being expressed as the fractal dimension $\left(D_{f}\right)$, were estimated for 11-22 cells from different flasks for every experiment and the significance of differences before and after OGD protocols was estimated by Student $t$ tests. The obtained data revealed that OGD protocols that lasted for up to $6 \mathrm{~h}$ did not cause a major reduction in cell viability. OGD protocols of $20 \mathrm{~min}$ and $1 \mathrm{~h}$ caused a significant reduction in SCMM ( $p<0.05$ and $p<0.01$, respectively), while there was no significant difference in SCMM values before and after the 3- and 6-h OGD protocols $(p>0.05)$. There was a significant increase in $\mathrm{A} / \mathrm{P}$ ratio after the 20 -min OGD protocol $(p<0.01)$, while a significantly decreased $\mathrm{A} / \mathrm{P}$ ratio $(p<0.05)$ and a significantly increased $\mathrm{D}_{\mathrm{f}}(p<$ 0.01 ) were revealed after the 3 -h OGD protocol. A significant increase in A/P ratio was revealed after the 6-h OGD protocol $(p<$ $0.01)$, while $\mathrm{D}_{\mathrm{f}}$ did not change significantly $(p>0.05)$. These results suggested that the 20 -min OGD protocol caused a reduction in pericyte movements and in the length of their processes, the 1-h OGD protocol caused a reduction in the movements, while the 3- and 6-h protocols did not affect these parameters. Thus, in this study we could not find evidence that pericytes die quickly when deprived of oxygen and nutrients, or that they remain contracted for a long period of time.

Prof. Zoran Redzic (Supervisor)

Dr. Andreas Henkel (Co-Supervisor) 